\section{Expression of some ATP-binding cassette transporters in acute myeloid leukemia}

\author{
Antonella Maria Salvia, ${ }^{1}$ \\ Flavia Cuviello, ${ }^{1}$ Sabrina Coluzzi, ${ }^{2}$ \\ Roberta Nuccorini, ${ }^{2}$ \\ Immacolata Attolico, ${ }^{2}$ \\ Sara Pasquina Pascale, ${ }^{2}$ \\ Faustino Bisaccia, ${ }^{1}$ Michele Pizzuti, ${ }^{2}$ \\ Angela Ostuni ${ }^{1}$ \\ ${ }^{1}$ Department of Sciences, University \\ of Basilicata, Potenza; ${ }^{2}$ Department of \\ Hematology, San Carlo Hospital, \\ Potenza, Italy
}

\begin{abstract}
Hematopoietic cells express ATP binding cassette $(\mathrm{ABC})$ transporters in relation to different degrees of differentiation. One of the known multidrug resistance mechanisms in acute myeloid leukemia (AML) is the overexpression of efflux pumps belonging to the superfamily of $\mathrm{ABC}$ transporters such as $A B C B 1, A B C G 2$ and $A B C C 1$. Although several studies were carried out to correlate $\mathrm{ABC}$ transporters expression with drug resistance, little is known about their role as markers of diagnosis and progression of the disease. For this purpose we investigated the expression, by real-time $\mathrm{PCR}$, of some ABC genes in bone marrow samples of AML patients at diagnosis and after induction therapy. At diagnosis, $A B C G 2$ was always down-regulated, while an up regulated trend for $A B C C 1$ was observed. After therapy the examined genes showed a different expression trend and approached the values of healthy subjects suggesting that this event could be considered as a marker of AML regression. The expression levels of some $\mathrm{ABC}$ transporters such as $A B C C 6$, seems to be related to gender, age and to the presence of FLT3/ITD gene mutation.
\end{abstract}

\section{Introduction}

The ATP Binding Cassette (ABC) is the largest transporter proteins superfamily which translocate a variety of substrates across the plasma membrane as well as across intracellular membranes of some organelles. The 49 human ABC genes are expressed in both normal and malignant cells and, among them, at least 14 are involved in transport of drugs and xenobi- otics. ${ }^{1}$ High expression of specific ABC transporter proteins contributes to multidrug resistance via the ATP-dependent drug efflux, a process that serves to protect cells from cytotoxic drugs causing failure of chemotherapy. $A B C B 1, A B C G 2$ along with $A B C C 1$ represent the three principal multidrug resistance genes that have been identified in tumor cells. ${ }^{2}$

ABC transporters besides being involved in drug resistance may contribute to the protection of undifferentiated human stem cells and of cells during initial tissue differentiation. Leukemia stem cells are characterized by a high expression of several $\mathrm{ABC}$ transporters ${ }^{3}$ and their down-regulation occurs upon differentiation. ${ }^{4}$ In addition, over-expression of certain ABC transporters in leukemic cells has been associated with major complication in the treatment of acute leukemia. ${ }^{5}$ In acute myeloid leukemia (AML) patients, multidrug resistance can be present intrinsically at diagnosis or can arise during chemotherapy as well as at relapse. ${ }^{6}$ Cancer treatment failure can be caused by MDR1 up-regulation, which was often associated with poor prognosis of AML. ${ }^{7,8}$ The ABCG2 gene was found overexpressed in patients with AML and it was associated with resistance to therapy. 9,10 Furthermore, the expression of $A B C G 2$ and $A B C B 1$ genes was detected in patients with AML with poor prognosis; ${ }^{11}$ in particular it was observed that $A B C B 1$ and the expression of $A B C G 2$ are prognostic factors in patients treated with daunorubicin and mitoxantrone but not with idarubicin. ${ }^{12}$

The involvement of the protein MRP1 $(A B C C 1)$ in chemoresistance of patients with AML is controversial. ${ }^{13}$ However, it was reported that, at the level of mRNA, MRP1 is overexpressed in patients with relapsed AML or who have not responded to treatment, compared with healthy controls or patients who have achieved complete remission. Moreover, the expression of this gene was correlated with some clinical characteristics, such as cytogenetic and FAB classifications. ${ }^{14}$

Therefore if these $\mathrm{ABC}$ transporters could play a role in drug resistance in AML, any correlation between MRP protein expression and clinical response in AML patients has been detected.

Few studies have been carried out on the involvement of the multidrug-resistance protein 6 (MRP6/ABCC6) in drug resistance. Among the molecules transported in vitro by MRP6, there are some drugs widely used in chemotherapy such as certain anthracyclines. ${ }^{15}$ In order to investigate the possible correlation of $\mathrm{ABC}$ transporters expression in AML diagnosis and response to drug treatments we studied mRNA
Correspondence: Angela Ostuni, Department of Sciences, University of Basilicata, Via dell'Ateneo Lucano, 85100 Potenza, Italy. Tel.: +39.0971.205453 - Fax +39.0971.205678. E-mail: angela.ostuni@unibas.it

Key words: Acute Myeloid Leukemia; Multidrug Resistance; $\mathrm{ABC}$ transporters; ABCC6.

Contributions: AMS performed real time PCR experiments, FC analyzed data, SC, RN and SPP prepared cell samples, IA and MP selected patients and discussed data, FB and $\mathrm{AO}$ designed the research and wrote the paper.

Conflict of interest: the authors declare no potential conflict of interest.

Received for publication: 13 September 2017 Accepted for publication: 18 October 2017.

This work is licensed under a Creative Commons Attribution-NonCommercial 4.0 International License (CC BY-NC 4.0).

(C) Copyright A.M. Salvia et al., 2017

Licensee PAGEPress, Italy

Hematology Reports 2017; 9:7406

doi:10.4081/hr.2017.7406

expression levels of $A B C C 6$ gene along with the expression of the well-known $A B C B 1, A B C C 1$ and $A B C G 2$ genes in bone marrow samples from 11 AML patients and from 7 healthy donors. The interest for $A B C C 6$ gene derives from the observation, reported in recent studies, that $A B C C 6$ is involved in the control of the cell cycle ${ }^{16}$ and regulates genes involved in the immune response. ${ }^{17}$ MRP6 is structurally and functionally poorly characterized ${ }^{18-21}$ mutations in this gene cause the Pseudoxanthoma elasticum, but its function and physiological substrate(s) are not known. ${ }^{22}$

There are some evidences about the possible involvement of epigenetic mechanisms regulating the expression of some $\mathrm{ABC}$ genes; ${ }^{23}$ it is known that histone deacetylase inhibitors (HDACis) and methyltransferase inhibitors cause upregulation of $A B C B 1$ and $A B C G 2 .{ }^{24-26}$ The effect of histone deacetylase and methyl transferase inhibitors on $A B C B 1$ and $A B C C 6$ expression was evaluated in AML cell line HL-60.

\section{Materials and Methods}

\section{Study setting}

In this retrospective study we included a total of 26 bone marrow samples, taken, after informed consent, from 11 adult AML 
patients (for eight of them there were both samples at the onset of the disease and after treatment) and 7 subjects without leukemia, who were seen between 2008 and 2012 at the Division of Hematology of the San Carlo Hospital, Potenza, Italy. We have excluded patients with acute promyelocytic leukemia. The study was performed in accordance with the Declaration of Helsinki. Patients were stratified by risk categories according to the criteria of the European LeukemiaNet. ${ }^{27}$ The percent of blasts in the bone marrow was detected with morphological examination by optical microscope. Cytogenetic investigations were carried out using conventional analysis and FISH; molecular analysis was done by qualitative PCR and real-time PCR.

Patients under 60 years of age received induction treatment with continuous infusion cytarabine (Ara-C) $100 \mathrm{mg} / \mathrm{m}^{2}$ for 7 days plus daunoblastin $60 \mathrm{mg} / \mathrm{m}^{2} /$ day for 3 days in infusion for 30 minutes; patients over 60 years of age received induction treatment in infusion with Ara-C $3 \mathrm{~g} / \mathrm{m}^{2}$ for 2 hours for 5 days plus idarubicin $45 \mathrm{mg} / \mathrm{m}^{2}$ in infusion for 1 hour on the fourth day.

Complete remission (CR) status was defined as normocellular marrow with marrow blasts $<5 \%$, absence of extramedullary disease, no blasts with Auer rods, normal peripheral blood counts and, independence from transfusion of red cells. Patients who did not fulfill the aforementioned criteria were defined as primary resistant (PR).

Subjects without AML were used as controls and compared to patients at diagnosis.

\section{Cell culture and in vitro treatment with Trichostatin A and 5-Aza- 2'deoxycytidine}

The HL60 cell line used in this study was purchased from DSMZ (www.dsmz.de) and maintained in RPMI-1640 (SigmaAldrich) culture medium, supplemented with $10 \%$ fetal bovine serum (EuroClone), $1 \%$ glutamine (EuroClone) and 1\% of the antibiotics penicillin and streptomycin (EuroClone) in polystyrene flasks at $37^{\circ} \mathrm{C}$ and $5 \% \mathrm{CO}_{2}$.

Cells were treated with $50 \mathrm{ng} / \mathrm{mL}$ or 500 ng/mL of Trichostatin A (TSA, Sigma Aldrich) or with $5 \mu \mathrm{M}$ or $50 \mu \mathrm{M}$ of 5 -Aza2 'deoxycytidine (Aza, Sigma - Aldrich) for 24 hours. Cells were harvested by centrifugation at $1200 \mathrm{rpm}$ for 5 minutes at $22^{\circ} \mathrm{C}$.

\section{RNA extraction, cDNA synthesis and quantitative real-time $\mathrm{PCR}$}

Total RNA was extracted from about $1 \times 10^{7}$ marrow cells or from $1 \times 10^{6}$ cultured cells using the GenElute Mammalian Total RNA Miniprep Kit (Sigma-Aldrich) accord- ing to the manufacturer's instructions and transcribed to cDNA using random primers and High Capacity cDNA Reverse Transcription Kit (Applied Biosystem). The following primers designed to span exonexon junctions to eliminate any undesirable genomic DNA amplification, were used: $A B C C 6$, forward 5'-TCGCTGTCTTCCTGAGCCTC-3' and reverse 5'CAT T T GC T C C T C C T GATGGT-3 '; $A B C G 2$, forward 5' ATCACTGATCCTTCCATCTTG-3' and reverse 5'-GCTTAGACATCCTTTTCAGG-3'; $A B C B 1$, forward 5'CCTTCAGGGTTTCACATTTGG-3' and reverse 5'-ACTCACATCCTGTCTGAGCA-3'; $A B C C 1$, forward 5'-GATCATGCTCACTTTCTGGC-3' and reverse 5'TGGGCAT C C T C T T T TAAGGC-3'; GAPDH, forward 5'-GATCATCAGCAATGCCTCCT-3' and reverse 5'-ACTGTGGTCATGAGTCCTTC-3'.

Level of gene transcripts was measured by real-time PCR amplification performed on a 7500 Fast Real Time PCR System (Applied Biosystems) using the Power SYBR Green PCR Master Mix (Applied Biosystems) for detection of PCR products. Analysis were performed in triplicate. The specificity of amplifications was confirmed by analyzing the melting curves. To evaluate the difference in the expression of target genes, the $\mathrm{C}_{t}$ (cycle threshold) of the targets was normalized with the $C_{t}$ of the endogenous control glyceraldehyde-3-phosphate dehydrogenase $(G A P D H)$. From obtained $\Delta \mathrm{C}_{\mathrm{t}}$ values, the relative expression level for each target PCR was calculated using the $\Delta \Delta \mathrm{C}_{\mathrm{t}}$ method.

\section{Statistical analysis}

Data were expressed as the mean \pm standard error (SE). Differences between any two groups were determined by Student's $t$ test; differences among more than two groups were determined by ANOVA. A Pearson product-moment correlation coefficient was computed to assess the relationship between gene expression and age, WBC count and \% BM blasts and between expression of drug resistance genes among themselves. A $\mathrm{P} \leq 0.05$ was considered statistically significant. The analysis was carried out using the statistical package $\mathrm{R}$ (version 3.1.3).

\section{Results}

To investigate the involvement of $A B C B 1, A B C C 1, A B C G 2$ and $A B C C 6$ transporters in the diagnosis and prognosis of AML, gene expression at the mRNA level has been determined in bone marrow sam-

Table 1. Demographic, clinical and hematological data of patients with acute myeloid leukemia.

\begin{tabular}{lc} 
Variables & Acute myeloid leukemia patients (n=11) \\
Age (years) & Median: 61 (range 37-81) \\
Gender & 6 \\
Males & 5 \\
Females & Median: 46.1 (range 3.56-223.7) \\
WBC count $\left(\times 10^{3}\right.$ cells $\left./ \mathrm{mm}^{3}\right)$ & Median: 36 (range 5.0-295) \\
\hline PLT count $\left(\times 10^{3}\right.$ cells $\left./ \mathrm{mm}^{3}\right)$ & Median: 80 (range 54-100) \\
\% Bone marrow blasts & 2 \\
\hline FAB morphology & 1 \\
M0 & 4 \\
M2 & 4 \\
M4 & \\
Not evaluable & 1 \\
Karyotype & 7 \\
Favorable & 1 \\
Intermediate (I e II) & 2 \\
Adverse & \\
Not evaluable & 4 \\
\hline FLT3 Status & 7 \\
WT & \\
Internal tandem duplication & 5 \\
NPM1 Status & $6(50 \%)$ \\
WT & \\
MUT & 5 \\
CR Status* (n=8) & \\
\hline
\end{tabular}

*Complete remission evaluated after induction chemotherapy. 
ples obtained at diagnosis of the disease and after chemotherapy. We analyzed samples from 11 patients with AML (six males and five females; median age $=61$ years, range $=37-81$ ), treated with cytarabine and anthracyclines, and samples from 7 healthy subjects (four males and three females; median age $=54$ years, range $=23-84$ ).

Clinical, laboratory and disease-specific characteristics of the 11 patients with AML are shown in Table 1. Among patients who received induction chemotherapy, 4 patients achieved a complete remission, 4 patients were primary resistant, two were non-evaluable due to death during induction and one patient has not undergone chemotherapy due to age.

Expression level of genes in AML and healthy samples are presented in Figure 1. In healthy controls, $A B C C 6$ gene showed expression levels lower than the other three genes (in terms of $\mathrm{C}_{\mathrm{t}}$, values were comprised between 9,66 and 18,2) while $A B C C 1$ has shown higher levels of expression than the others; $A B C B 1$ and $A B C G 2$ showed similar and low expression levels. In AML patients at diagnosis, expression levels of $A B C C 6$ and $A B C B 1$ were similar to those found in the controls (in terms of $\mathrm{C}_{\mathrm{t}}$, values resulted between 5.83 and 15.88 and between 6.74 and 13.88 , respectively). Conversely, $A B C C 1$ showed higher levels compared to the controls: it was significantly overexpressed in $73 \%$ of patients $(\mathrm{P}=0.019)$. Instead, $A B C G 2$ was always down-regulated in AML patients at diagnosis $(\mathrm{P}=0.0004)$, compared with controls.

Analysis to evaluate the potential interrelationship of these genes with clinical variables (such as some prognostic factors) of patients with AML has been performed (Table 2). We have observed a correlation between expression levels of genes and the age of patients: older patients presented lower expression of $A B C B 1, A B C C 1$ and $A B C C 6$ than younger ones $(\mathrm{P}<0.05)$. $A B C C 6$ and $A B C C 1$ expression levels resulted also related to gender $(\mathrm{P}<0.05)$, in particular we have observed that female patients presented expression levels higher than males. The expression levels of $A B C C 6$ and $A B C C 1$ were also higher in AML samples positive for FLT3/ITD gene mutation $(\mathrm{P}<0.05)$ than in the negative ones, while the level of $A B C G 2$ was higher in samples negative for the same mutation $(\mathrm{P}<0.01)$ than in the positive ones. No significant association was observed between gene expression levels and other clinical variables.

mRNA levels of genes have been also analyzed after drug treatment (induction therapy) of 8 patients and compared to those of healthy subjects and of patients at diagnosis of the pathology (Figure 2). After the chemotherapy treatment $A B C G 2$ showed expression levels higher than at diagnosis $(\mathrm{P}=0.012) . A B C C 1(\mathrm{P}=0.47)$ and $A B C B 1(\mathrm{P}=0.48)$ gene expressions showed a downward trend in most of the patients after treatment compared to the diagnosis. Instead, no variation was observed in $A B C C 6$ gene expression $(\mathrm{P}=0.77)$.

It has been reported that some $\mathrm{ABC}$ genes are transcriptionally controlled by epigenetic mechanisms. To evaluate the effect of Trichostatin A (an inhibitor of histone deacetylase) and 5-Aza-2'deoxycytidine (an inhibitor of DNA methyltransferase) on the $A B C C 6$ gene expression, realtime PCR analysis was performed in the AML cell line HL-60. As a control, we eval-

\section{Discussion}

It is known that $\mathrm{ABC}$ transporters are the major causes of drug resistance in both solid and hematological cancers. Leukemia stem cells are characterized by a high expression of several $\mathrm{ABC}$ transporters, ${ }^{3}$ above all P-glycoprotein $(A B C B 1), \mathrm{MRP} 1$ $(A B C C 1)$ and BCRP $(A B C G 2)$ whose role in multidrug resistance has often been con-

Table 2. Correlation between expression of drug resistance genes with clinical variables of patients with acute myeloid leukemia.

\begin{tabular}{lcccc} 
Clinical and demographic variables & $\mathrm{ABCB} 1$ & $\mathrm{ABCG}$ & $\mathrm{ABCC}$ & $\mathrm{ABCC6}$ \\
Age & $0.03^{*}$ & 0.94 & $0.05^{*}$ & $0.047^{*}$ \\
Gender & 0.87 & 0.66 & $0.04^{*}$ & $0.03^{*}$ \\
\hline WBC $\left(\times 10^{3} \mathrm{cell} s / \mathrm{mm}^{3}\right)$ & 0.95 & 0.36 & 0.14 & 0.69 \\
\% BM blasts & 0.82 & 0.73 & 0.23 & 0.43 \\
\hline Karyotype & 0.31 & 0.15 & 0.91 & 0.71 \\
FLT3 Status & 0.51 & $0.009^{*}$ & $0.03^{*}$ & $0.03^{*}$ \\
\hline NPM1 Status & 0.55 & 0.25 & 0.39 & 0.57 \\
\hline
\end{tabular}

*The values in the table indicate the P-values obtained as described in the Statistical Analysis section.

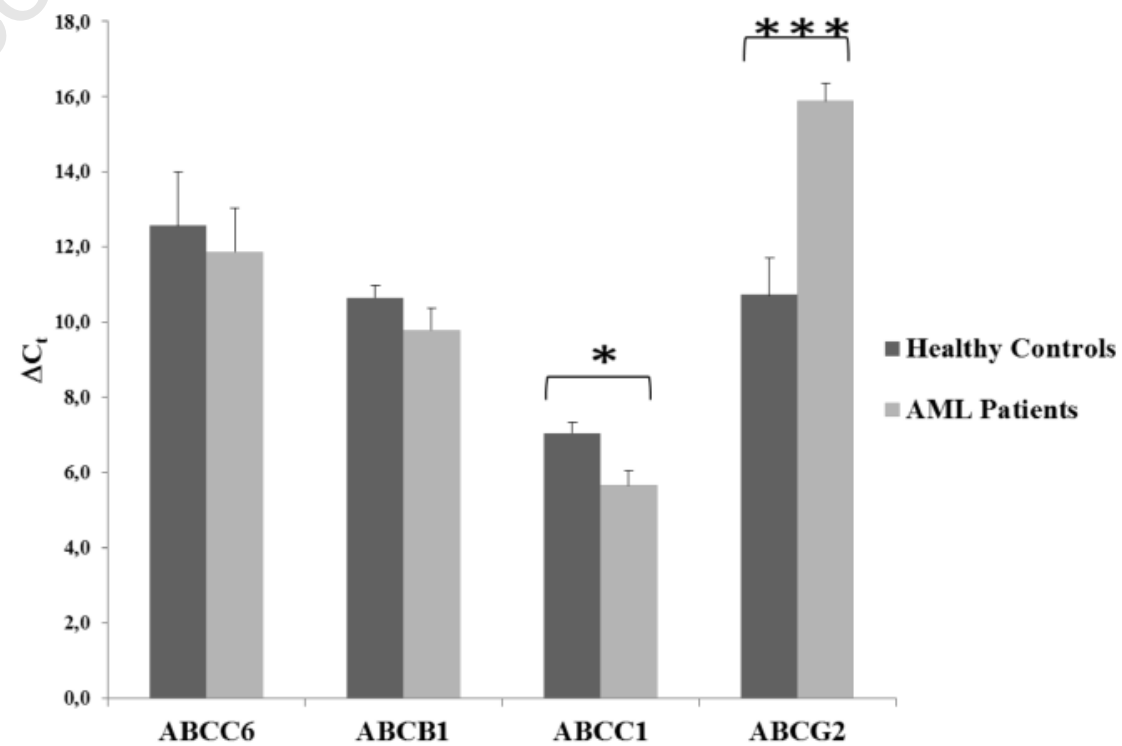

Figure 1. Expression level of $A B C C 6, A B C B 1, A B C C 1$ and $A B C G 2$ genes at diagnosis in subjects with (AML Patients, $n=11$ ) and without (Healthy Controls, $n=7$ ) AML. $\Delta C_{t}$ values were obtained by $\left[C_{t}\right.$ target gene $-C_{t}$ GAPDH $]$. Results are represented as the mean $\pm S$.E., ${ }^{*} \mathbf{P}<\mathbf{0 . 0 5}$; ${ }^{* * *} \mathbf{P}<\mathbf{0 . 0 0 1}$. 
tradictory.9-13 Only few studies have addressed the role of other ABC transporters and no data have been reported for possible involvement in AML of MRP6 ( $A B C C 6)$ which has been proven to affect the cell cycle and genes expression. ${ }^{16,17}$ In this study we investigate the correlation of mRNA levels of some ABC transporters in bone marrow samples obtained from healthy donors and AML patients, at diagnosis and after treatment with a combination of Ara-C and either daunoblastin or idarubicin.
Results showed that at diagnosis $A B C G 2$ gene is always down-regulated and $A B C C 1$ is always up-regulated compared to healthy donors, while no correlation was observed between $A B C B 1, A B C C 6$ and AML. mRNA analysis of samples from AML patients after drug treatment indicated that $A B C B 1$ and $A B C C 1$ tend to be downregulated and $A B C G 2$ is almost always overexpressed. Therefore, $A B C G 2$ increases and $A B C C 1$ tends to decrease, bringing their expression values averagely closer to those of healthy subjects, showing that

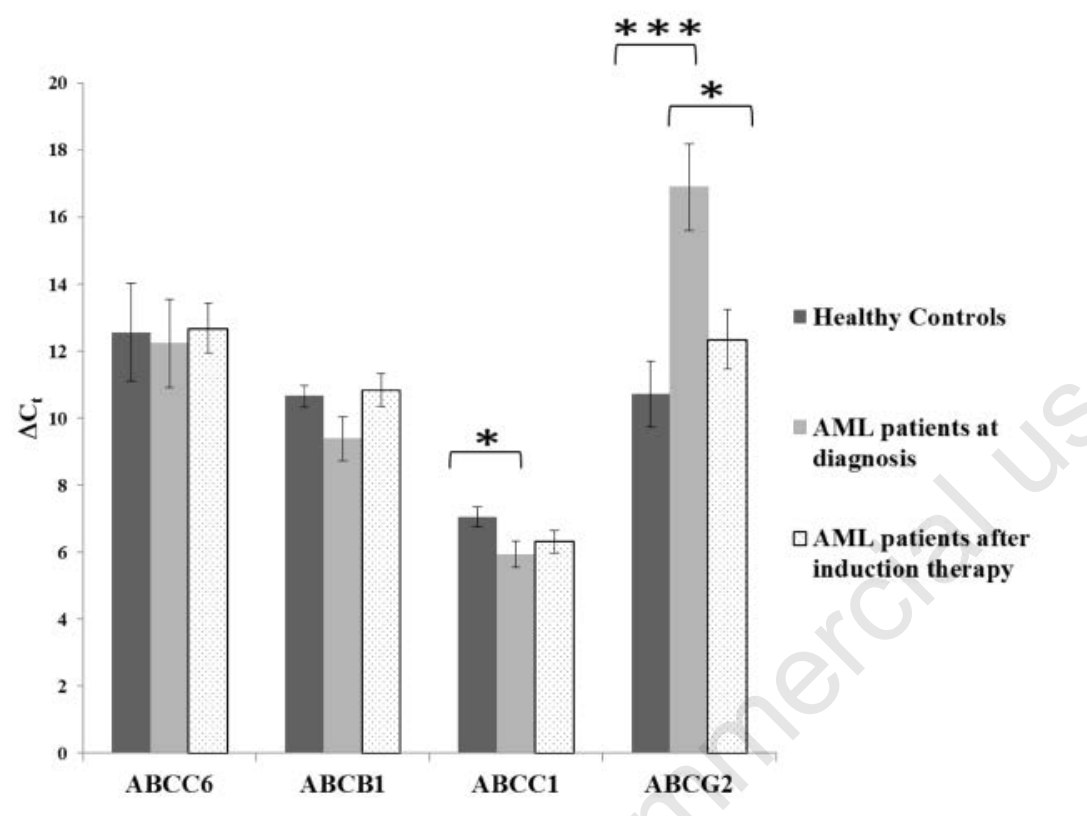

Figure 2. Expression level of $A B C C 6, A B C B 1, A B C C 1$ and $A B C G 2$ genes in AML patients $(n=8)$, at the diagnosis of the pathology and after induction therapy, and in healthy controls. $\Delta C_{t}$ values were obtained by $\left[C_{t}\right.$ target gene $-C_{t}$ GAPDH $]$. Results are represented as the mean \pm S.E, ${ }^{*} \mathbf{P}<0.05$.

A

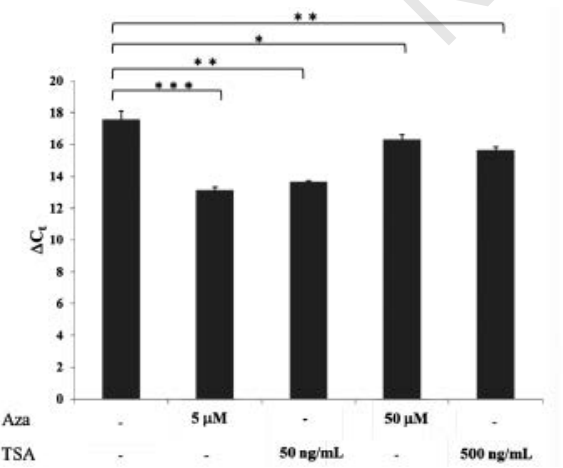

B

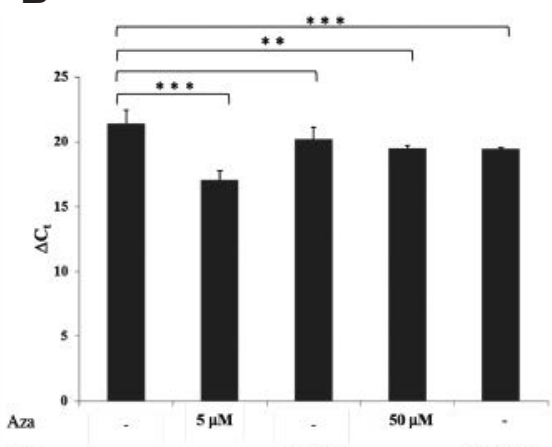

Figure 3. Expression level of ABCC6 (A) and ABCB1 (B) genes in HL-60 cell line treated with 5-Aza-2'deoxycytidine $(5 \mu \mathrm{M}$ or $50 \mu \mathrm{M})$ or with Trichostatin $A(50 \mathrm{ng} / \mathrm{mL}$ or 500 $\mathrm{ng} / \mathrm{mL}) . \Delta \mathrm{C}_{t}$ values were obtained by $\left[\mathrm{C}_{t}\right.$ target gene $-C_{t}$ GAPDH $]$. Error bars represent the Standard Error (S.E.) of the mean for three independent experiments. ${ }^{*} \mathbf{P}<0.05$; ${ }^{* *} \mathbf{P}<0.01 ; * * * \mathbf{P}<0.001$.

these transporters, above all $A B C G 2$, are closely correlated with AML or rather that their expression may be correlate to the disease and may represent markers of therapeutic response.

We have observed a correlation between mRNA levels of some genes and the age of AML patients: older patients presented lower expression of $A B C B 1, A B C C 1$ and $A B C C 6$ than younger ones. $A B C C 1$ and $A B C C 6$ mRNA levels showed a correlation with gender (female patients presented expression levels higher than males) and with the FLT3/ITD gene mutation (patients positive for this mutation showed overexpression of these genes).

The 5-Aza-2'deoxycytidine and Trichostatin A experiments on cell culture confirm the epigenetic control for these genes.

\section{Conclusions}

Although our study was conducted on a small set of patients, it has shown that a transcription analysis of a small set of differentially expressed $\mathrm{ABC}$ genes might be useful not only for AML diagnosis ( $A B C G 2$ was always down-regulated, while an up regulated trend for $A B C C 1$ was observed) but also to follow the progression of the disease (both transcripts tend to approach the values of healthy subjects after induction therapy). Further studies on a larger cohort of patients should be performed to confirm the results reported here and might provide further information on the possible role of $A B C C 6$ in drug resistance induced by epigenetic drugs used in the treatment of patients with AML and on its correlation with gender, age and presence of FLT3/ITD gene mutation.

\section{Reference}

1. Dean M, Rzhetsky A, Allikmets R. The human ATP-binding cassette (ABC) transporter superfamily. Genome Res 2001;11:1156-66.

2. El-Awady R, Saleh E, Hashim A, et al. the role of eukaryotic and prokaryotic $\mathrm{ABC}$ transporter family in failure of chemotherapy. Front Pharmacol 2016;7: 535.

3. Grouw EP, Raaijmakers MH, Boezeman JB, et al. Preferential expression of a high number of ATP binding cassette transporters in both normal and leukemic CD34+. Leukemia 2006;20:750-4.

4. Huls M, Russel FG, Masereeuw R. The role of ATP binding cassette trans- 
porters in tissue defense and organ regeneration. J Pharmacol Exp Ther 2009;328:3-9.

5. Steinbach $\mathrm{D}$ and Legrand $\mathrm{O}$. $\mathrm{ABC}$ transporters and drug resistance in leukemia: was P-gp nothing but the first head of the Hydra? Leukemia 2007;21:1172-6.

6. Damiani D, Tiribelli M, Calistri E, et al. The prognostic value of Pglycoprotein $(\mathrm{ABCB})$ and breast cancer resistance protein $(\mathrm{ABCG} 2)$ in adults with de novo acute myeloid leukemia with normal karyotype. 2006;91:825-8.

7. Pallis M, Turzanski J, Higashi Y, Russell N. P-glycoprotein in acute myeloid leukaemia: therapeutic implications of its association with both a multidrug-resistant and an apoptosisresistant phenotype. Leuk Lymphoma 2002;43:1221-8.

8. Wuchter C, Leonid K, Ruppert V, et al. Clinical significance of P-glycoprotein expression and function for response to induction chemotherapy, relapse rate and overall survival in acute leukemia. Haematologica 2000;85:711-21.

9. Galimberti S, Guerrini F, Palumbo GA, et al. Evaluation of BCRP and MDR-1 co-expression by quantitative molecular assessment in AML patients. Leuk Res 2004;28:367-72.

10. Damiani D, Tiribelli M, Calistri E, et al. The prognostic value of P-glycoprotein $(\mathrm{ABCB})$ and breast cancer resistance protein (ABCG2) in adults with de novo acute myeloid leukemia with normal karyotype. Haematologica 2006;91:825-8.

11. Marzac C, Garrido E, Tang R, et al. ATP Binding cassette transporters associated with chemoresistance: transcriptional profiling in extreme cohorts and their prognostic impact in a cohort of 281 acute myeloid leukemia patients.
Haematologica 2011;96:1293-301.

12. Benderra Z, Faussat AM, Sayada L, et al. Breast cancer resistance protein and P-glycoprotein in 149 adult acute myeloid leukemias. Clin Cancer Res 2004;10:7896-902.

13. Van der Pol MA, Broxterman HJ, Pater $\mathrm{JM}$, et al. Function of the ABC transporters, P-glycoprotein, multidrug resistance protein and breast cancer resistance protein, in minimal residual disease in acute myeloid leukemia. Haematologica 2003;88:134-47.

14. Mahjoubi F, Golalipour M, Ghavamzadeh A, Alimoghaddam K. Expression of MRP1 gene in acute leukemia. Sao Paulo Med J 2008;126:172-9.

15. Belinsky MG, Chen ZS, Shchaveleva I, et al. Characterization of the drug resistance and transport properties of multidrug resistance protein 6 (MRP6, ABCC6). Cancer Res 2002;62:6172-7.

16. Miglionico R, Ostuni A, Armentano $\mathrm{MF}$, et al. ABCC6 knockdown in HepG2 cells induces a senescent-like cell phenotype. Cell Mol Biol Lett 2017 22:7.

17. Miglionico R, Armentano MF, Carmosino M, et al. Dysregulation of gene expression in ABCC6 knockdown HepG2 cells. Cell Mol Biol Lett 2014;19:517-26.

18. Ostuni A, Miglionico R, Monné M, et al. The nucleotide-binding domain 2 of the human transporter protein MRP6. J Bioenerg Biomembr 2011;43:465-71.

19. Ostuni A, Miglionico R, Castiglione Morelli MA, Bisaccia F. Study of the nucleotide-binding domain 1 of the human transporter protein MRP6. Prot Pept Lett 2010;17:1553-58.

20. Miglionico R, Gerbino A, Ostuni A et al. New insights into the roles of the Nterminal region of the ABCC6 transporter. J Bioenerg Biomembr
2016;48:259-67.

21. Cuviello F, Tellgren-Roth Å, Lara P, et al. Membrane insertion and topology of the amino-terminal domain TMD0 of multidrug-resistance associated protein 6 (MRP6). FEBS Lett 2015;589:39218.

22. Hendig D, Langmann T, Kocken S, et al. Gene expression profiling of $\mathrm{ABC}$ transporters in dermal fibroblasts of pseudoxanthoma elasticum patients identifies new candidates involved in PXE pathogenesis. Lab Invest 2008;88:1303-15.

23. Arrigoni E, Galimberti S, Petrini M, et al. ATP-binding cassette transmembrane transporters and their epigenetic control in cancer: an overview. Exp Opin Drug Metabol Toxicol 2016;12:1419-32.

24. Bram EE Stark M, Raz S, Assaraf YG. Chemotherapeutic drug-induced ABCG2 promoter demethylation as a novel mechanism of acquired multidrug resistance. Neoplasia 2009;11:1359-70.

25. Hauswald S, Duque-Afonso J, Wagner $\mathrm{MM}$, et al. Histone deacetylase inhibitors induce a very broad, pleiotropic anticancer drug resistance phenotype in acute myeloid leukemia cells by modulation of multiple $\mathrm{ABC}$ transporter genes. Clin Cancer Res 2009;15:3705-15.

26. Calcagno AM, Fostel JM, To KK, et al. Single-step doxorubicin-selected cancer cells overexpress the ABCG2 drug transporter through epigenetic changes. Br J Cancer 2008;98:1515-24.

27. Döhner H1, Estey EH, Amadori S, et al. European LeukemiaNet. Diagnosis and management of acute myeloid leukemia in adults: recommendations from an international expert panel, on behalf of the European LeukemiaNet. Blood 2010;115:453-74. 